\title{
Image quality analysis of an antique microscope objective with a Shack-Hartmann wavefront sensor: an experiment of educational interest
}

\section{Análisis de la calidad de imagen de un objetivo de microscopio antiguo mediante un sensor de frente de onda Shack-Hartmann: un experimento de interés educativo}

\author{
A. Marzoa ${ }^{1,2,3, S^{*}}$ and S. Vallmitjana ${ }^{3, S}$ \\ 1. Departament de Física, Escola d'Enginyeria de Telecomunicació i Aeroespacial de Castelldefels (EETAC), \\ Universitat Politècnica de Catalunya, C/ d'Esteve Terradas, 7, 08860 Castelldefels, Spain \\ 2. SENER Aeroespacial, S.A., Parc de l'Alba, C/Creu Casas i Sicart, 86-87, 08290 Cerdanyola del Vallès, Spain \\ 3. Departament de Física Aplicada, Facultat de Física, Universitat de Barcelona, C/ de Martí i Franquès, 1, 11, \\ 08028 Barcelona, Spain
}

(") E-mail: antonio.marzoa@upc.edu

S: miembro de SEDOPTICA / SEDOPTICA member

Received: 19/04/2021 Accepted: 11/06/2021

DOI: 10.7149/OPA.54.2.51062

\begin{abstract}
:
In this work we report the image quality analysis of an antique microscope objective from ca 1850 by using a non-contact technique based on a Shack-Hartmann wavefront sensor. The method and the experiment are described, and results are fully discussed. The technique provides useful and interesting tools for colour image formation process simulations. Application of this work for educational purposes is also commented.
\end{abstract}

Key words: wavefront sensing, Shack-Hartmann, aberrations, microscope, education, image processing

\section{RESUMEN:}

En este trabajo presentamos el análisis de la calidad de imagen de un objetivo de microscopio antiguo datado ca 1850 mediante una técnica basada en el uso de un sensor de frente de onda Shack-Hartmann. Se describen la base del método utilizado y los experimentos realizados, y los resultados se describen para probar la validez de dicho método. La técnica presentada y utilizada en este trabajo supone una herramienta útil e interesante para simular el proceso de formación de imágenes en color. Se comenta, también, el potencial uso del trabajo con fines educativos.

Palabras clave: análisis del frente de onda, Shack-Hartmann, aberraciones, microscopio, educación, procesado de imagen

\section{REFERENCES AND LINKS / REFERENCIAS Y ENLACES}

[1] E. Abbe, Beiträge zur Theorie des Mikroskops un der Mikroskopischen wahrnehmung, Archiv für Mikroskopische Anatomie 9, 413-468 (1873).

[2] L. Rayleigh, On the theory of optical images, with special references to the microscope, Phil. Mag. 46 (5), 167 (1896).

[] M. I. Cross, M. J. Cole, Modern Microscopy. A handbook for Beginners and Students. Chicago, Chicago Medical Book Company (1912).

[4] A. van Helden, The Invention of the Telescope. Phliadelphia, The American Philosophical Society (1977). 
[5] B. K. Johnson, Optics and Optical Instruments. New York, Dover Publications, Inc. (1960).

[6] M. Born, E. Wolf, Principles of optics: Electromagnetic theory of propagation, interference and diffraction of light. Cambridge, Cambridge University Press (1999).

[7] W. J. Smith, Modern Optical Engineering. New York, McGraw-Hill (1998).

[8] J. W. Goodman, Introduction to Fourier Optics. Berkshire, McGraw-Hill (1998).

[9] F. Zernike, Beugungstheorie des Schneidenverfahrens und Seiner Verbesserten Form, der Phasenkontrastmethode, Physica 1 (8) 689-704 (1934).

[10] S. Vallmitjana, C. Ferran, S. Bosch, Non-contact technique for testing antique optical instruments based on wavefront sensing. Journal of Modern Optics, 58:14, 1269-1277 (2010).

[11] R. C. Gonzalez, R. E. Woods, Digital Image Processing, Pearson Educational (2008).

[12] B. C. Platt, R. Shack, History and Principles of Shack-Hartmann Wavefront Sensing. Journal of Refractive Surgery, 17, 5, 573-577 (2001).

[13] R. W. Duffner, R. Q. Fugate, The Adaptive Optics Revolution: A History. New Mexico, University of New Mexico Press (2009).

[14] J. Liang, B. Grimm, S. Goelz, J. F. Bille, Objective measurement of wave aberrations of the human eye with the use of a Hartmann-Shack wave-front sensor. J. Opt. Soc. Am., 11 (7), 1949-1957 (1994).

[15] C. López-Quesada, J. Andilla, E. Martín-Badosa, Correction of aberration in holographic optical tweezers using Shack-Hartmann sensor. App. Optics, 48 (6), 1084-1090 (2009).

[16] L. Lakshminarayanan, Zernike polynomials: a guide. Journal of Modern Optics, 58, 545-561 (2011).

[17] V. J. Mahajan, Aberration Theory made simple. Washington, SPIE PRESS Tutorial Text (1991).

[18] R. J. Noll, Zernike polynomials and atmospheric turbulence. J. Opt. Soc. Am., 66 (3), 207-211 (1976).

[19] E. P. Goodwin, J. C. Wyant, Interferometric Optical Testing, Washington, SPIE PRESS Field Guide Series (2006).

[20] J. A. Díaz-Navas, V. Mahajan, Orthonormal aberration polynomials for optical systems with circular and annular sector pupils. J. Opt. Soc. Am., 66 (3), 207-211 (1976).

[21] S. Vallmitjana, Instrumentos científicos. Catálogo de la Facultad de Física de la Universidad de Barcelona. Barcelona, Publicacions i edicions Universitat de Barcelona (2011).

[22] https://www.lomography.com/magazine/328315-charles-chevalier-and-the-story-of-a-lens-legacy

[23] http://www.ub.edu/museuvirtual/index en.php

[24] Y. Zhang, H. Gross, Systematic design of microscope objectives. Part I: System review and analysis. Adv. Opt. Techn., 8 (5), 313-347 (2019).

[25] Y. Zhang, H. Gross, Systematic design of microscope objectives. Part II: Lens modules and design principles. Adv. Opt. Techn., 8 (5), 349-384 (2019).

[26] http://www.fush.lv/Kingbright/Data/KAF-5060PBESEEVGC\%28V7\%29.pdf

[27] https://www.theimagingsource.com/products/industrial-cameras/firewire-400-color/dfk31bf03h/

[28] S. Bará, E. Palios, J. Arines, Signal-to-noise ratio and aberration statistics in ocular aberrometry. Opt. Lett., vol. 37 (12), 2427-2429 (2012).

[29] G. Prieto, A CDMAM Image Phantom software improvement for human observer assessment. Tesis doctoral, Madrid, Universidad Complutense de Madrid (2009).

[30] Z. Wang, A. C. Bovik, H. R. Sheikh, E. P. Simoncelli, Image Quality Assessment: From Error Visibility to Structural Similarity. IEEE Transactions on Image Processing, vol. 13, no. 4 (2004).

[31] A. Carnicer, I. Juvells, S. Vallmitjana, S. Bosch, Processament Òptic i Digital d'Imatges. Barcelona, Edicions i Publicacions de la Universitat de Barcelona (2001).

[32] V. Greco, G. Molesini, F. Quercioli, Telescopes of Galileo. Applied Optics, 32:31, 6219-6226 (1993).

[33] S. Vallmitjana, I. Juvells, J. R. de Francisco Moneo, S. Bosch, F. Abbad, A. Carnicer, F. X. Monzonís, M. Montes-Usategui and J. Ferré-Borrull, Pràctiques de Laboratori d'Òptica. Textos Docents. Publicacions i Edicions de la Universitat de Barcelona. Barcelona (2005).

[34] J. Pérez-Tudela, Análisis de la influencia de las aberraciones del Sistema difractor en el reconocimiento de imágenes por correlación óptica. Tesis doctoral, Barcelona, Universitat de Barcelona (2006).

[35] A. Marzoa, S. Vallmitjana, S. Bosch, Wavefront measurements of imaging systems by comparing a PointDiffraction Interferometer and a Shack-Hartmann wavefront sensor. Opt. Pura Apl., 51 (2) 50027:1-10 (2018). 


\section{Introduction}

Instrumental Optics achieved great successes when, in between the late XVIII and during XIX centuries, the huge problem of optical aberrations started to be compensated [1], [2]. As an example, of the relevance of those developments, there is a long list of discoveries in the fields of Biology and Astronomy [3], [4] in that period. This makes it a period of great interest for the study of the development of Instrumental and Experimental Optics and Optical Design [5], [6], [7] and many related disciplines.

For that reason, we propose the analysis of the optical quality of and instrument belonging to such era, in order to introduce and motivate students in optics specialization to the fields of wavefront sensing and image quality, while it could also be a potential tool for making them aware of the importance of the conservation of those instruments.

In this work we present an interesting experiment which reviews different aspects related to the Instrumental Optics such as the formulation of the image formation process [6], [7], [8], image quality and aberrations, Shack-Hartmann wavefront sensor basis, Fourier Optics [8] and Zernike polynomials [9]. Some basic and common image processing techniques are also described and used in the analysis.

The paper first presents a review on the theoretical background of the topic related to the experiment, after that, the method is introduced, and the experimental set-up is described. Finally, the results are presented, and teaching opportunities and validity of the method are discussed.

The method used in this study was previously applied to a terrestrial optical telescope [10]. Now we extend the technique to the analysis of an antique microscope objective and discuss its applicability for educational purposes.

\section{Theoretical background}

This section reviews the whole theoretical basis necessary for the implementation of the method.

\section{2.a. Image formation process}

From the geometrical point of view, any kind of optical system can be modelled as a black-box defined by two planes: the entrance and the exit pupil (see Figure 1) [8], [10]. Let $U_{o}\left(x_{0}, y_{0}\right)$ be the complex amplitude distribution that defines a certain object in the plane $\left(x_{0}, y_{0}\right)$ (as indicated in Figure 1). For a linear and spatial invariant instrument, the so-called Gaussian, geometric or "perfect" image of the object, in the plane $\left(x_{i}, y_{i}\right)$, is written as:

$$
U_{g}\left(x_{i}, y_{i}\right)=\frac{1}{|\beta|} U_{o}\left(\frac{x_{0}}{\beta} \cdot \frac{y_{0}}{\beta}\right)
$$

Being $\beta$ the total magnification introduced by the instrument.

In the case of a diffraction-limited intensity invariant optical system, the "real" image can be written as the convolution of the geometric image with the so-called incoherent Point-Spread Function (PSF) [6]:

$$
U_{i}\left(x_{i}, y_{i}, z_{i}\right)=\iint U_{g}\left(x^{\prime}, y^{\prime}\right) \cdot\left|h\left(x-x^{\prime}, y-y^{\prime}, z_{i}\right)\right|^{2} d x^{\prime} d y^{\prime}=U_{g}(x, y) *|h(x, y, z)|^{2}
$$

Where $h\left(x, y, z_{i}\right)$ is the complex amplitude of the incoherent PSF [6], [8], which can be computed in the Fresnel diffraction framework as follows:

$$
h(x, y, z) \propto \frac{1}{\lambda z} \int_{E x P} \exp \left[i k W\left(\xi_{2}, \eta_{2}\right)\right] \cdot \exp \left[i k \frac{\Delta z}{2 z^{2}}\left(\xi_{2}^{2}+\eta_{2}^{2}\right)\right] \cdot \exp \left[i \frac{k}{z}\left(x \xi_{2}+y \eta_{2}\right)\right] d \xi_{2} d \eta_{2}
$$

Where $k=2 \pi / \lambda$ is the wavenumber and $W\left(\xi_{2}, \eta_{2}\right)$ corresponds to how the optical system deteriorates the image of the object in the process, i.e. the amount of the aberration introduced in the image by the optical 
system (see section 2.c. for a further description of that element). The second exponential term refers to defocusing, i.e. to the propagation term of the Fresnel diffraction, and the last term is just the kernel of the Fourier Transform [8].

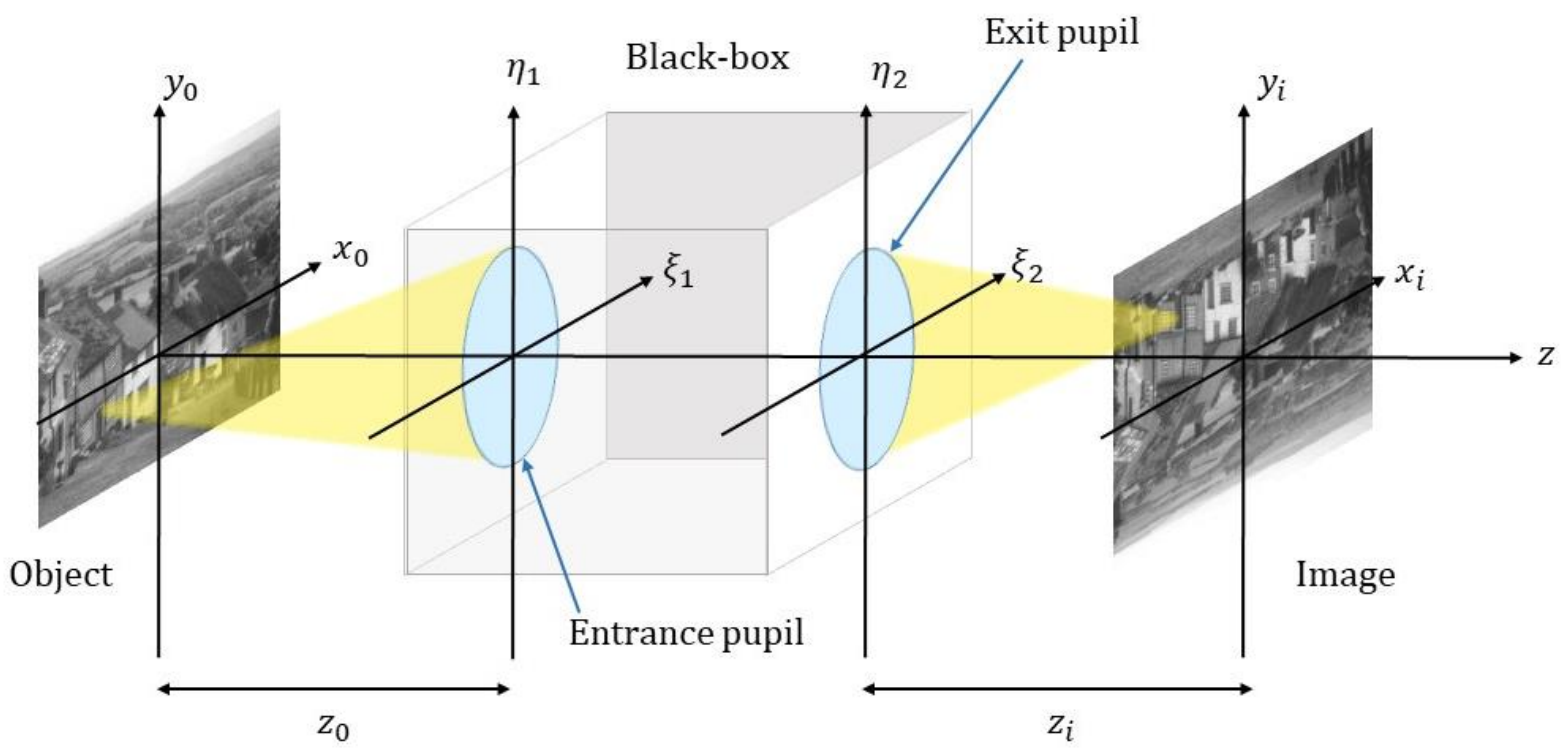

Figure 1. Schematic drawing of a black-box optical system. Nomenclature introduced in this figure is used in all the mathematical formalism of that section.

\section{2.b. Polychromatic description}

The wave aberration function $W(x, y)$ and the PSF are defined as monochromatic concepts. To treat it for polychromatic images, i.e. for the case of RGB images, as it is the case in this study, we should treat the aberration function for each wavelength independently.

Thus, under isoplanatism ${ }^{1}$ [8] assumption, the Fourier analysis previously described for monochromatic sources, can be extended to the polychromatic case with considering a colour sensitive receiver. Within this framework, we consider the decomposition of a polychromatic image into the RGB values, which is a good approximation for the register process made by colour charge-coupled devices (CCD) [11]. Thus, we get:

$$
I_{i}^{C}(x, y)=\int_{\lambda} I_{i}(x, y, \lambda) S(\lambda) d \lambda
$$

Where $I_{i}^{C}(x, y)$ is the intensity distribution captured by the camera, $I_{i}(x, y, \lambda)$ is the intensity distribution for a certain $\lambda$ and $S(\lambda)$ is the spectral weight or response of the receptor.

It is commonly assumed that $S(\lambda)$ is assimilated in three filters: $R(\lambda), G(\lambda)$ and $B(\lambda)$. Thus, for CCD cameras with three separated colour filters we get:

$$
\begin{aligned}
& I_{R}^{C}(x, y)=\int_{\lambda} I^{C}(x, y, \lambda) R(\lambda) d \lambda \\
& I_{G}^{C}(x, y)=\int_{\lambda} I^{C}(x, y, \lambda) G(\lambda) d \lambda
\end{aligned}
$$

\footnotetext{
${ }^{1}$ Isoplanatism is a noun derived from the term isoplanatic, which refers to a shift invariant property of the imaging system. Sometimes the word is directly used for meaning such as "same aberrations" over the system.
} 


$$
\begin{gathered}
\text { ÓPTICA PURA Y APLICADA } \\
\frac{\text { www.sedoptica.es }}{I_{B}^{C}(x, y)=\int_{\lambda} I^{C}(x, y, \lambda) B(\lambda) d \lambda}
\end{gathered}
$$

Which provides equivalent results in the case of CCD cameras with Bayer filters [10]. The range of integration corresponds to the visible, i.e., the values for $\lambda$ range from 400 to $700 \mathrm{~nm}$.

If we denote the corresponding gaussian images as: $I_{R_{g}}^{C}, I_{G_{g}}^{C}$ and $I_{B_{g}}^{C}$, and do the same considerations for the PSFs, we get:

$$
\begin{aligned}
& I_{R_{i}}^{C}(x, y)=I_{R_{g}}^{C} * P S F_{R} \\
& I_{G_{i}}^{C}(x, y)=I_{G_{g}}^{C} * P S F_{G} \\
& I_{B_{i}}^{C}(x, y)=I_{B_{g}}^{C} * P S F_{B}
\end{aligned}
$$

And then, the image $I_{i}^{C}(x, y)$ is obtained.

\section{2.c. Shack-Hartmann wavefront sensor and optical aberrations}

The Shack-Hartmann (SH) wavefront sensor (also known as Hartmann-Shack in the literature) [12] is a commonly well-known and extended instrument for the analysis of the quality of a wavefront generated by a particular optical system, such as a telescope or the human eye, and has applications in a broad set of different scientific and technical topics [13], [14], [15].

An SH sensor basically consists of a lenslet array and a photon detector (for instance, a CCD camera). If an ideal non-aberrated plane wavefront reaches the microlenses array, each lens focuses a perfect diffractionlimited spot in a particular point of the detector, defined by the optical axis of the microlens. Moreover, if the wavefront presents some aberrations, the spot will be formed in a different location. Thus, with a suitable software able to measure the displacement of the spot from the ideal position, it is possible to quantify the amount of aberration of the incident wave (see Figure 2).

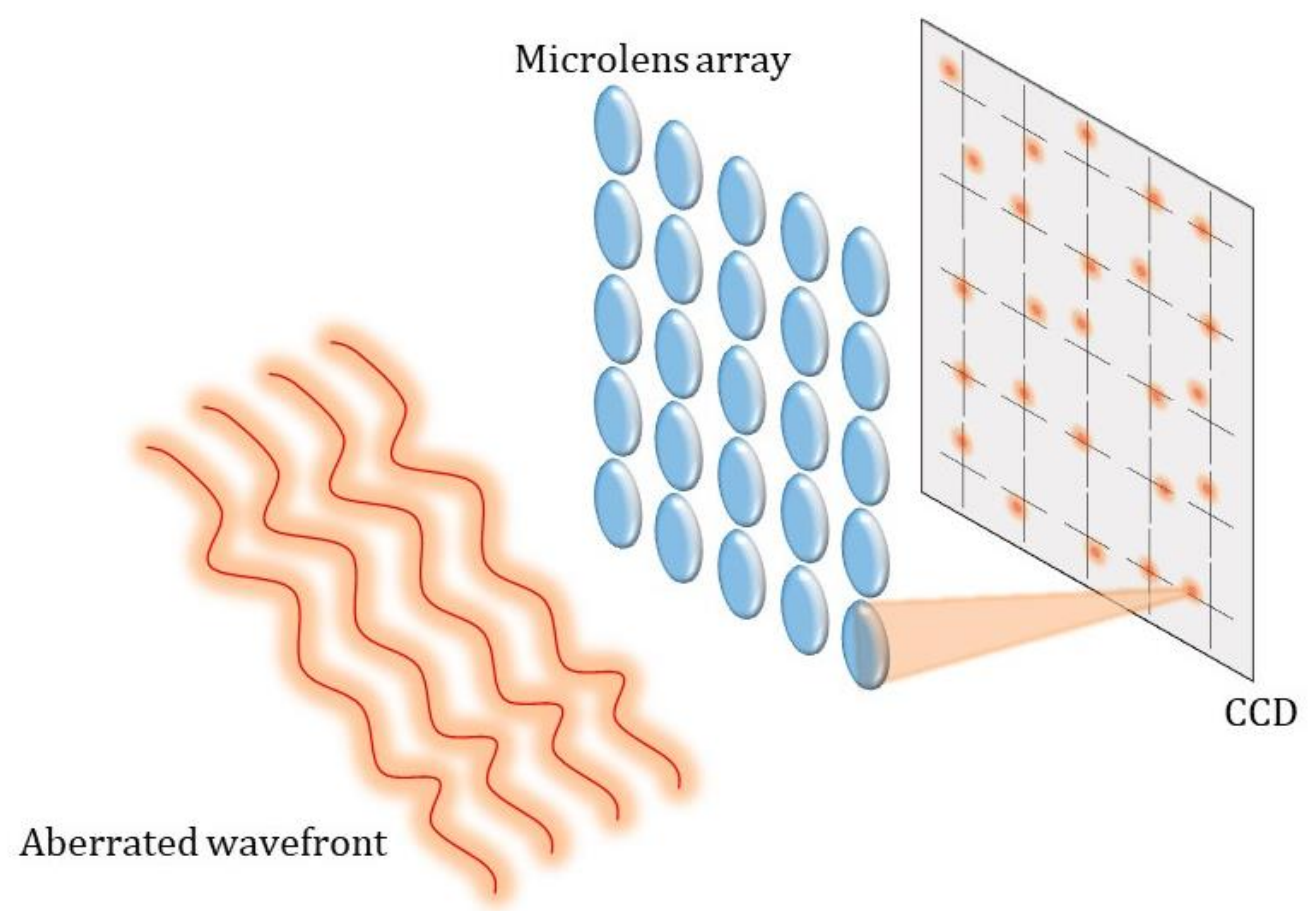

Figure 2. Basic sketch of an SH: an aberrated wavefront reaches the lenslet array and it segments the incident wave in order to measure the deviation of the actual spot from the ideal situation. 
There are several ways to express the corresponding measured aberrations. A commonly used approach is to express the aberration in terms of Zernike polynomials. Those set of polynomials were first introduced by the Nobel Laureate in Physics Fritz Zernike ${ }^{2}$ (1888 - 1966) in 1934 [9], and are a spread tool used for wavefront representation.

Zernike polynomials are orthonormal and continuous over the unit circle [9], [16], making them a perfect set of candidates for describing aberrations of common circular-aperture optical instruments ${ }^{3}$ [17]. The aberration polynomial $W(\rho, \theta)$ in polar coordinates can be expressed in terms of Zernike polynomials as follows:

$$
W(\rho, \theta)=\sum_{n=0}^{\infty} \sum_{m=0}^{n} C_{n}^{m} Z_{n}^{m}(\rho, \theta)
$$

Where $C_{n}^{m}$ denotes the Zernike coefficient refereed to a certain term $Z_{n}^{m}(\rho, \theta)$. In our study, we restrict to the first nine terms ${ }^{4}$, thus, equation (7) can be simplified as follows:

$$
\begin{gathered}
W(\rho, \theta)=C_{0}^{0}+C_{1}^{1} \rho \cos \theta+C_{1}^{-1} \rho \sin \theta+C_{2}^{0}\left(2 \rho^{2}-1\right)+C_{2}^{-2} \rho^{2} \sin (2 \theta)+C_{2}^{2} \rho^{2} \cos (2 \theta) \\
+C_{3}^{-1}\left(3 \rho^{3}-2 \rho\right) \sin \theta+C_{3}^{1}\left(3 \rho^{3}-2 \rho\right) \cos \theta+C_{4}^{0}\left(6 \rho^{4}-6 \rho^{2}+1\right)
\end{gathered}
$$

In this expression, the first coefficient corresponds to the piston term, whereas the two that follow are the tilts (tilt $0^{\circ}$ and tilt $90^{\circ}$, respectively). The other terms are (in order of appearance in the expression): focus, astigmatism at $0^{\circ}$, astigmatism at 45으.

\section{Description of the experiment}

The main objective of that section is to describe the method and set-up to analyse the instrument under testing.

The idea is to measure the wavefront generated by the antique objective with a SH sensor for three different wavelengths, in order to obtain the PSF for each colour and convolve them with the three different channels of a "perfect" RGB image, obtaining the simulated image created by the instrument.

\section{3.a. The instrument under test}

The instrument chosen for the experiment is a microscope objective taken from a microscope from the Collection of Scientific instruments [21] of the Faculty of Physics of the Universitat de Barcelona, UB, (Figure 3), dated ca 1850. In the Faculty's archive there is a handwritten instruction manual dated 1880 with the manufacturer's official seal 5 .

The objective appertains to the French brand Chevallier. This brand was created by the optician and instrument maker Charles Louis Chevallier (1804 - 1859), who worked on the construction of lenses and objectives for the daguerreotype and the camera lucida [23]. We reefer the interested reader to [24] and [25] references for a general approach on microscope objectives designs.

\footnotetext{
2 Fritz Zernike received the Nobel Prize in Physics in 1934 for the invention of phase contrast microscope.

${ }^{3}$ As it is explained in the text, Zernike polynomials are a common tool, however there are different representations for them. Furthermore, Zernike polynomials are not the unique way of representing wavefronts. For a deeper understanding and investigation of this topic, the interested author is referred to [16], [17], [18], [19] and [20].

${ }^{4}$ The reasons for that choice are discussed later.

5 More information of this instrument can be found in its corresponding Virtual Museum [22] page (item 132, signature FFUB-0044): http://tempesta.media/mvub/es/Coleccion/instrumentos-cientificos/.
} 
The microscope presents a circular plate where the slide samples can be placed, and it is illuminated through a mirror. The instrument and its different components are saved in a wooden box.

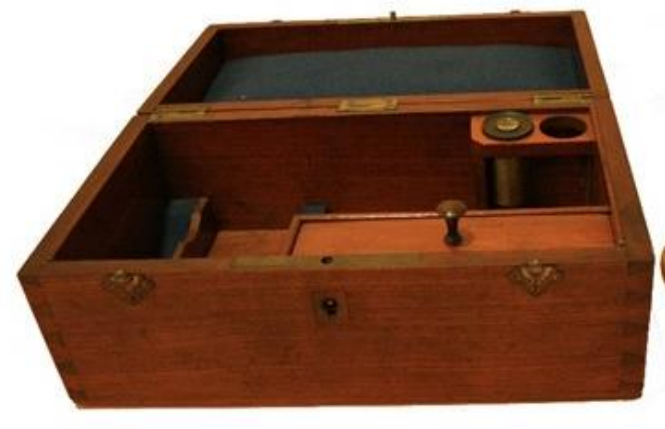

a)

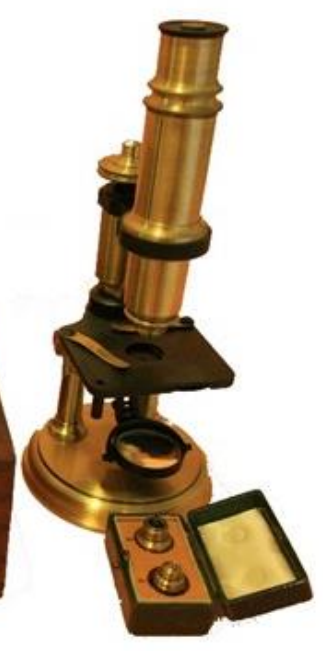

b)

Figure 3. The microscope (left) and detail of the objective under test (right).

\section{3.b. Experimental set-up}

As illumination source we used three different LED bulbs (KAF-5060PBESEEVGC) whose main wavelengths were: $621 \mathrm{~nm}$ (red, R), $525 \mathrm{~nm}$ (green, G) and $470 \mathrm{~nm}$ (blue, B) [26]. The size of each LED bulb is about 216 $\mu \mathrm{m}$ so, to generate a "punctual" source, a commercial $12 \times$ magnification highly corrected microscope objective was used to form an image of the LED bulb of $18 \mu \mathrm{m}$. To correctly align the LED bulbs with the optical axis, they were mounted in an $x-y-z$ micrometric stage. This configuration permit to choose the illumination, i.e., the colour, for each measurement with accuracy.

For the measurement of the wavefront, a commercial HASO32 ${ }^{\mathrm{TM}} \mathrm{SH}$ sensor from the Imagine Optic Company was used. This instrument consists of an array of $32 \times 32$ microlenses in front of a $512 \times 512$ pixels CCD sensor. The precision of the apparatus is of the order of $\lambda / 100$. The software supplied with the device permits to perform a Zernike modal reconstruction, allowing the measurement of Zernike coefficients.

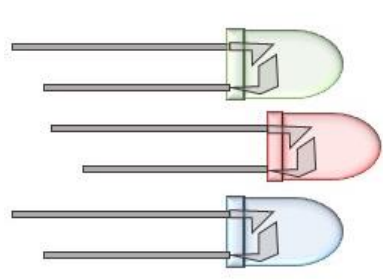

LED source

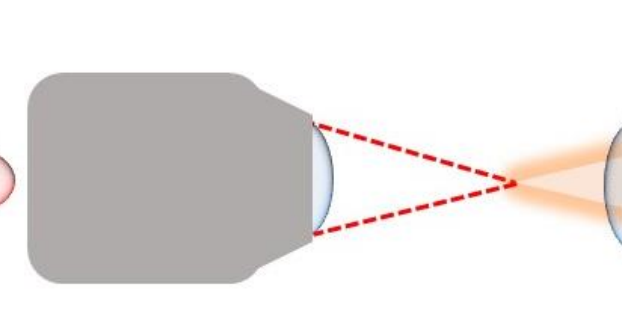

$12 \times$ microscope objective

Antique objective

Figure 4. Not-to-scale schematic experimental set-up: a tricolour light source compound of three different LED is imaged by a modern microscope objective, creating a point source that is placed at the front-focal-plane of the antique microscope objective under test. Finally, the actual wavefront generated by the instrument is recorded by the $\mathrm{SH}$ wavefront sensor. 


\section{3.c. Description of the software}

After obtaining the experimental values of the Zernike coefficients, they were introduced in our own software written in MALTAB(C) (The Mathworks, Inc., Natick, Massachusetts (US)). The software first computes $W(\rho, \theta)$ using (8), for each wavelength and then, using (3) the PSF is obtained. It is important to point out here that the PSF was always computed at the best plane $z$ for each wavelength, i.e. in the plane which corresponds to the focal position for each wavelength.

Then, by separating in three channels the reference image (see section 3.d.), the convolution between each channel and the PSF for each wavelength is performed (using (6)). By adding the three channels together again, the simulated image, as it should be seen through the Chevallier's objective is obtained. Figure 5 shows a block diagram of the software scheme and the method.

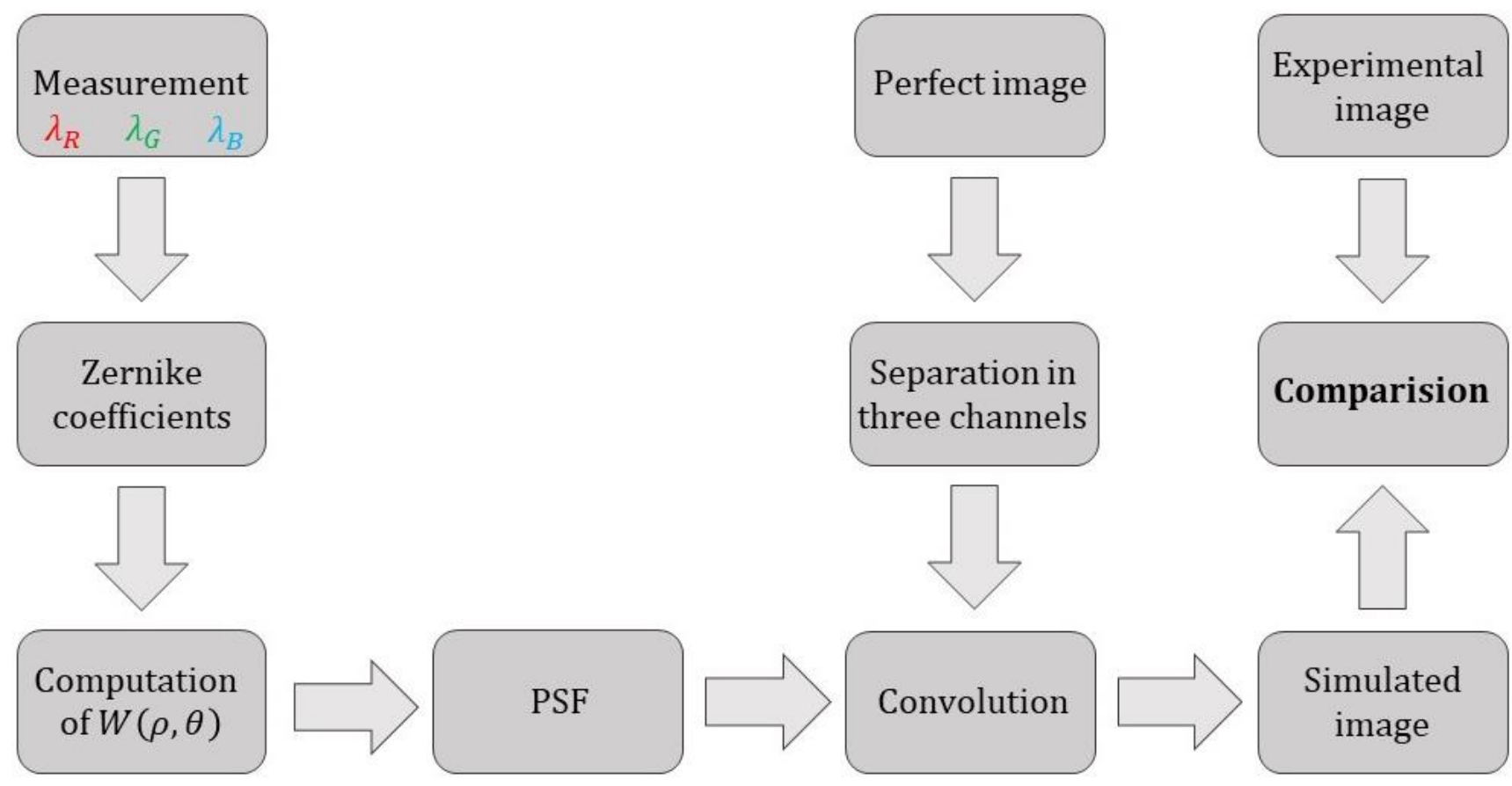

Figure 5. Block diagram of the method used in this work.

\section{3.d. Reference and experimental images}

To obtain a "perfect" reference image, a modern 20x and 0.4 NA microscope objective from PZO® was used. This objective presented similar optical parameters to the Chevallier's (i.e., similar focal length and field) but with a better optical quality.

The experimental images with this new objective were taken using a 768x768 pixel CCD camera from The Imaging Source(C) (specifications of the camera can be found in page 8 of [27]) for the case of six different specimens (see Table 1). The image of the same microscopic samples was also captured using the Chevallier objective for final results comparison purposes.

TABLE 1. Identification of the samples used in the study to get reference images.

\begin{tabular}{|c|c|}
\hline \hline Sample number & Specimen \\
\hline \hline 1 & Diatomea Arachnoidiscus Erenbergeli \\
2 & Diatomea Actinoptychus Heliopelta (in B1 451) \\
4 & Diatomea Triceratium Favus \\
5 & Cut of a Graaf's follicle from cat ovary \\
6 & Cut of an arm of a sea star Asteria Rubens \\
\hline
\end{tabular}




\section{Results}

With the set-up described in Figure 4, several measurements of the wavefront generated for each wavelength by the antique microscope objective were done. Taking into account that the most relevant aberrations for a system such the one analysed in this study are, despite the piston and tip-tilt coefficients, the first six Zernike terms [24], [25], and the fact that, for that particular case, adding more polynomial terms does not add relevant or significant information ${ }^{6}$, the final measurements were restricted for the first nine coefficients. Results of Zernike coefficients for each wavelength are shown in Table 2. The reconstruction of the wavefront and the corresponding PSF for each wavelength are presented in Figure 6.

TABLE 2. Value of the eight first Zernike coefficients (in microns) for the three sets of lambdas (in $\mathrm{nm}$ ). Piston is always excluded.

\begin{tabular}{|c|c|c|c|}
\hline \hline$\lambda(\mathrm{nm})$ & 621 & 525 & 470 \\
\hline \hline$C_{1}^{1}$ & -1.6779 & -5.2505 & -4.8357 \\
$C_{1}^{-1}$ & 2.9190 & 2.9358 & 8.9615 \\
$C_{2}^{0}$ & 22.3584 & 32.1063 & 41.6198 \\
$C_{2}^{-2}$ & 0.0375 & 0.0270 & 0.3097 \\
$C_{2}^{2}$ & -0.1940 & -0.2984 & -0.4381 \\
$C_{3}^{-1}$ & 0.0463 & 0.0154 & -0.1145 \\
$C_{3}^{1}$ & -0.0408 & -0.5537 & -0.8008 \\
$C_{4}^{0}$ & 0.2222 & 0.4489 & 0.3381 \\
\hline
\end{tabular}

$\mathrm{R}$
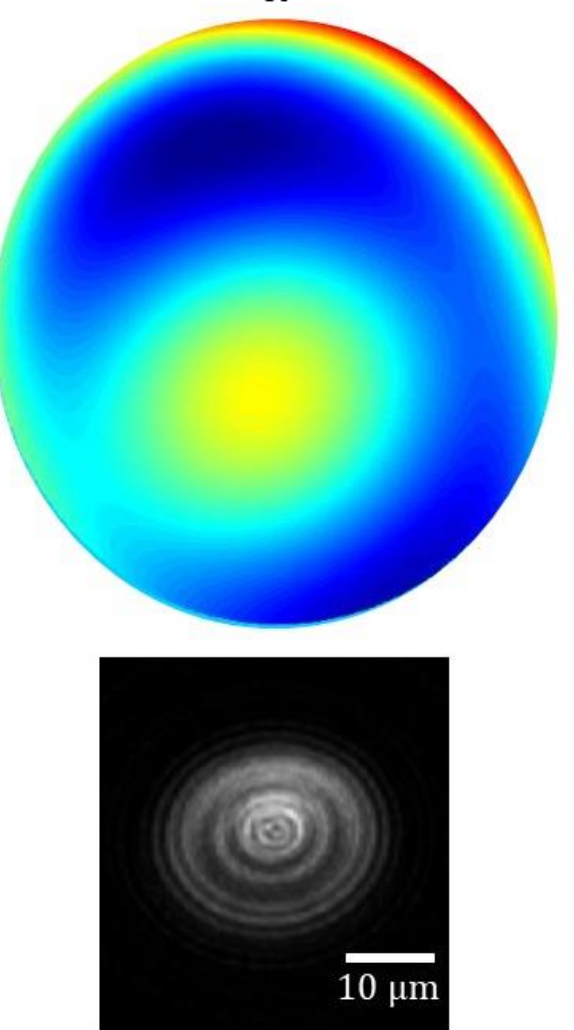

G
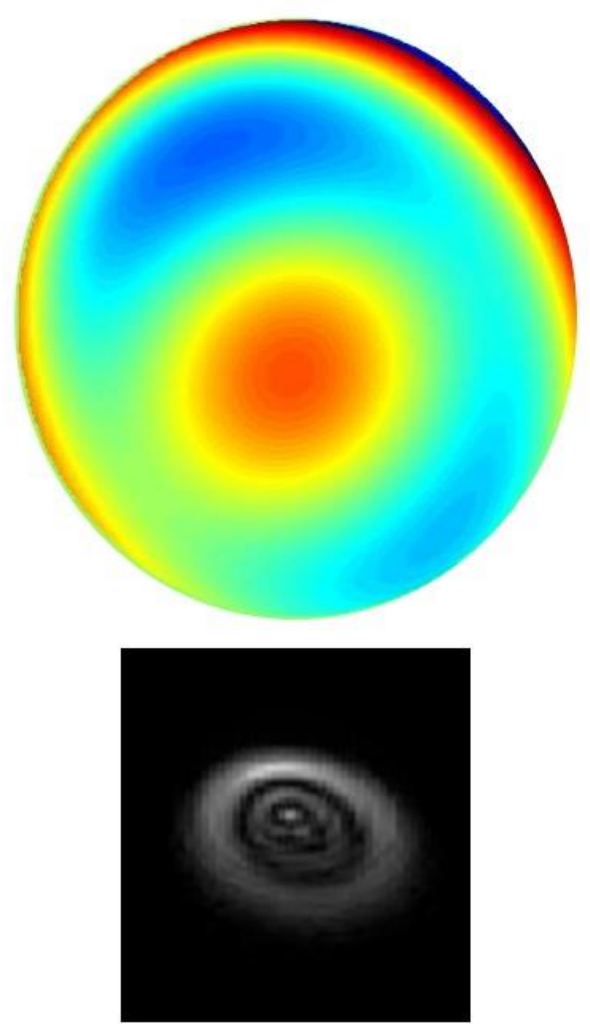

B

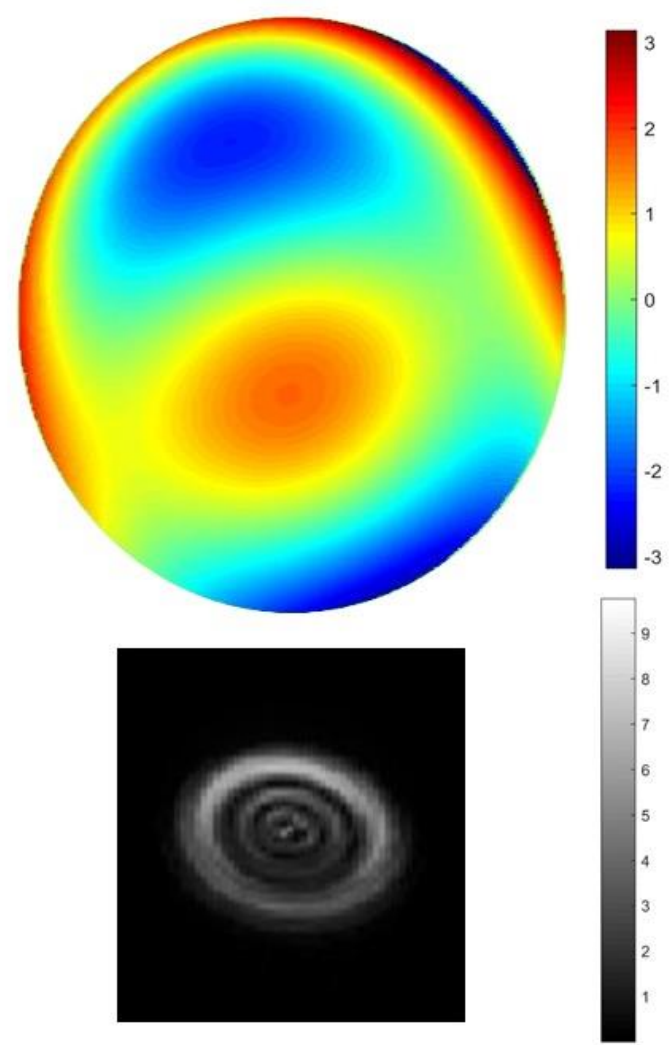

Figure 6. Reconstruction of the wavefront for each wavelength, excluding the first four Zernike terms, and the corresponding PSF. The colorbar is in lambda units whereas the grey bar reefers to the grey-level scale of the PSF images, in which the 0 reefers to the 0 and black pixel value and 10 to the 255 white pixel value.

\footnotetext{
${ }^{6}$ The discussion on the adequate number of Zernike coefficients on wavefront reconstruction is a huge and interesting topic out of the scope of this paper. The interested reader may refer to [28] for a rapid and interesting discussion. For the case analysed in this work, as in the case reported in [10], and as it is shown in [24] and [25], the first nine terms provide the representation of the most relevant aberrations.
} 
Figure 7 shows the set of reference images (see Table 1), the simulated ones, and the real images obtained with the Chevalier objective. A visual inspection shows that the simulated images are always similar to the real ones.

\section{Reference image Simulated image Chevalier's real image}

Sample 1
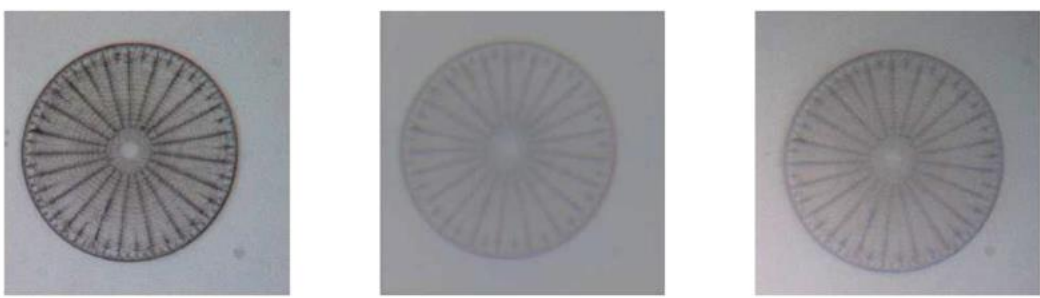

Sample 2
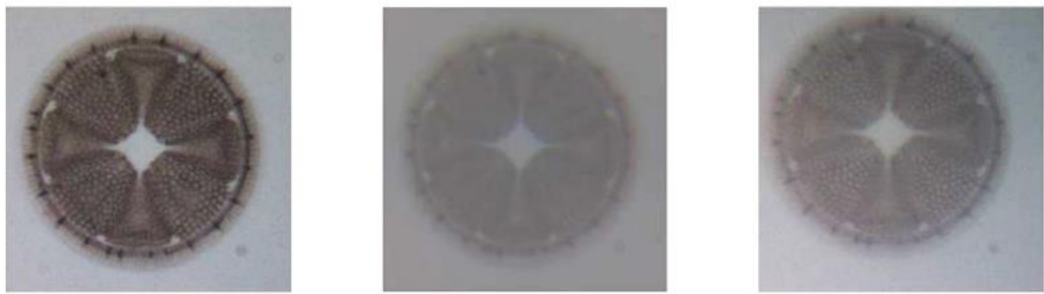

Sample 3
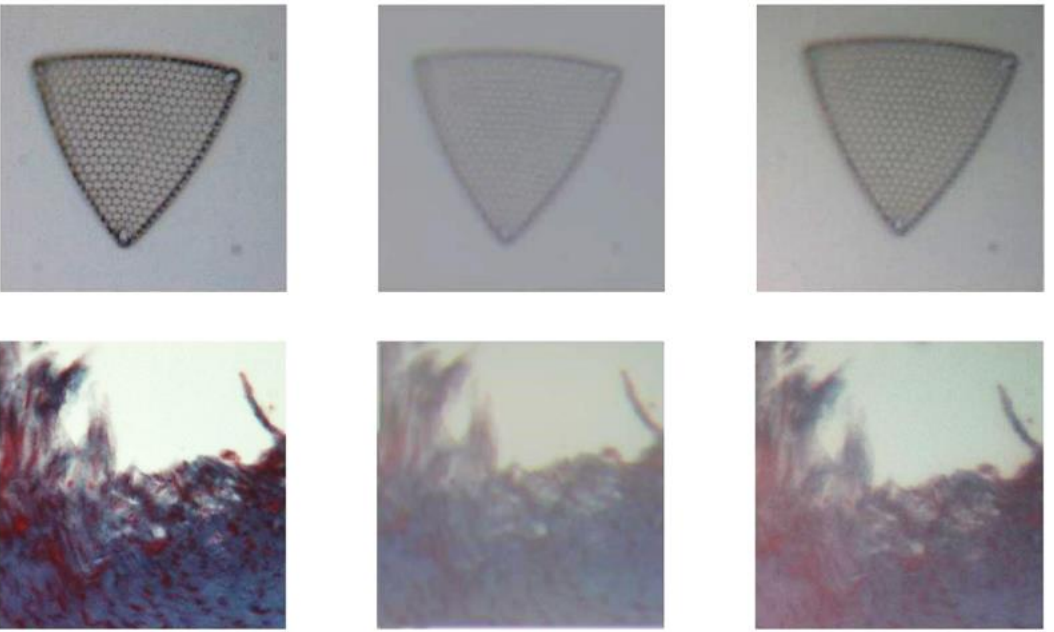

Sample 4
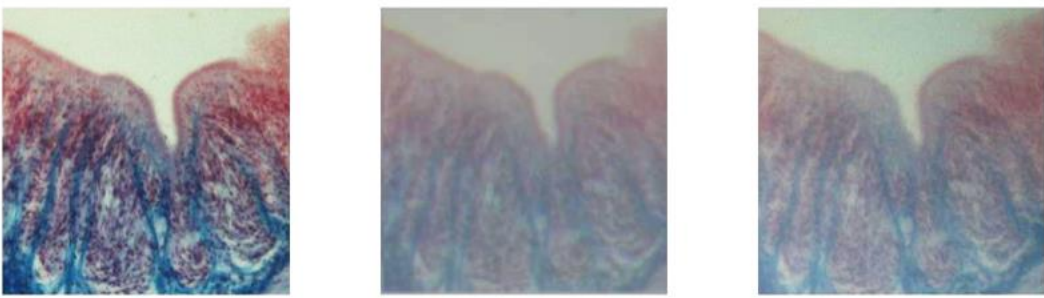

Sample 5
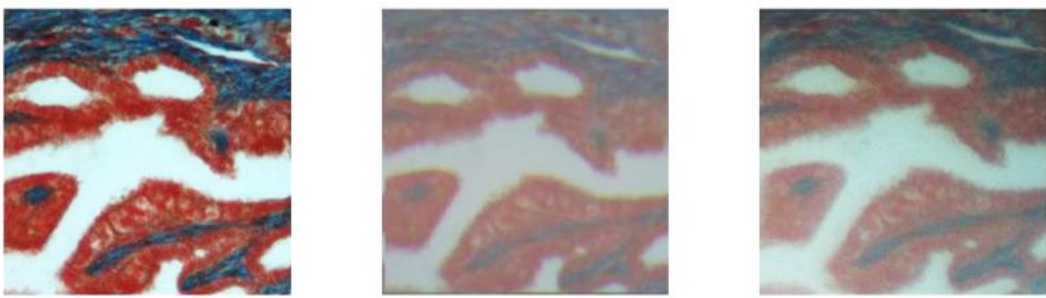

Figure 7. Results of the experiment: first column corresponds to the "perfect" reference images obtained with the PZO objective and the CCD camera, the second corresponds to the results of the simulation and the third column shows the images obtained using the antique microscope objective. Rows refer to each sample. 
Furthermore, to quantify the potential and validity of the technique previously presented, a simple digital test was performed. Figure 8 shows the results of such test, which is based on the application of Structural Similarity Index (SSIM) [29], [30].

SSIM is a common perceptual index used to evaluate the degradation of an image because of the application of post-processing strategies. The closer to 1 the value is of the SSIM between two images, the more similar they are. It is important to point out that it is essential that both images have the same size, orientation, and the same number of pixels for a proper use of the SSIM.

Let $X$ and $Y$ be two different images that are supposed to be compared. Let $\boldsymbol{x}=\left\{x_{i} \mid i=1,2, \ldots, N\right\}$ and $\boldsymbol{y}=$ $\left\{y_{i} \mid i=1,2, \ldots, N\right\}$ be two different areas of the images $X$ and $Y$, respectively, and $\mu_{x}$ and $\mu_{y}$ the mean pixel values of $\boldsymbol{x}$ and $\boldsymbol{y}$, and $\sigma_{x}$ and $\sigma_{y}$ the corresponding typical deviations.

Thus, the mean values are directly related with the mean luminance $l(x, y)$ value of the image through the mathematical relation:

$$
l(x, y)=\frac{2 \mu_{x} \mu_{y}+C_{1}}{\mu_{x}^{2}+\mu_{y}^{2}+C_{1}}
$$

Where $C_{1}$ is a constant used to stabilize the division when the denominator is quite small.

In a similar way, the contrast $c(x, y)$ of the image is defined, in terms of the parameters introduced above, as follows:

$$
c(x, y)=\frac{2 \sigma_{x} \sigma_{y}+C_{2}}{\sigma_{x}^{2}+\sigma_{y}^{2}+C_{2}}
$$

Where $C_{2}$ is a constant defined by the same purposes of $C_{1}$ in (9).

Finally, taking the covariance $\sigma_{x y}$, it is possible to define the structure index $s(x, y)$ as follows:

$$
s(x, y)=\frac{\sigma_{x y}+C_{3}}{\sigma_{x} \sigma_{y}+C_{3}}
$$

And, in this case, $C_{3}$ is a constant also defined to deal with instabilities when the denominator is close to zero.

Using equations (9), (10) and (11) it is possible to define the SSIM as the following combination:

$$
\operatorname{SSIM}(x, y)=l(x, y)^{\alpha} \cdot c(x, y)^{\beta} \cdot s(x, y)^{\gamma}
$$

Where $\alpha, \beta$ and $\gamma$ are the free weights used to add relevance to a certain component of equation (12). Setting the weight parameters to 1 , it is possible to rewrite (12) as follows:

$$
\operatorname{SSIM}(x, y)=\frac{\left(2 \mu_{x} \mu_{y}+C_{1}\right)\left(2 \sigma_{x y}+C_{2}\right)}{\left(\mu_{x}^{2}+\mu_{y}^{2}+C_{1}\right)\left(\sigma_{x}^{2}+\sigma_{y}^{2}+C_{2}\right)}
$$

Thus, we took the MATLAB ${ }^{7}$ function $\operatorname{ssim}($ ), which directly computes (12), and obtained the results shown in Figure 8 and Table 3.

\footnotetext{
${ }^{7}$ MATLAB® is a registered trademark from The MathWorks Inc@.
} 
ÓPTICA PURA Y APLICADA

www.sedoptica.es

$\mathrm{R}$

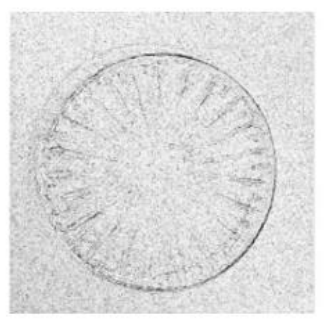

Sample 1

Sample 2
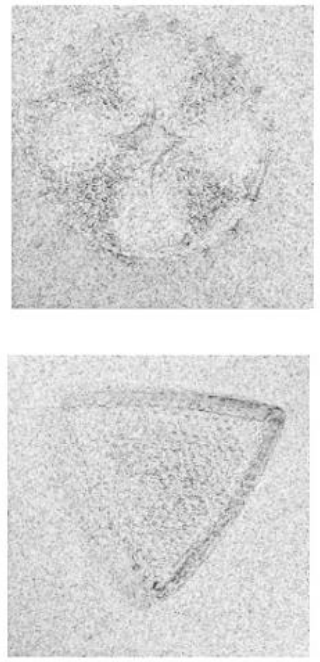

Sample 3

Sample 4

Sample 5
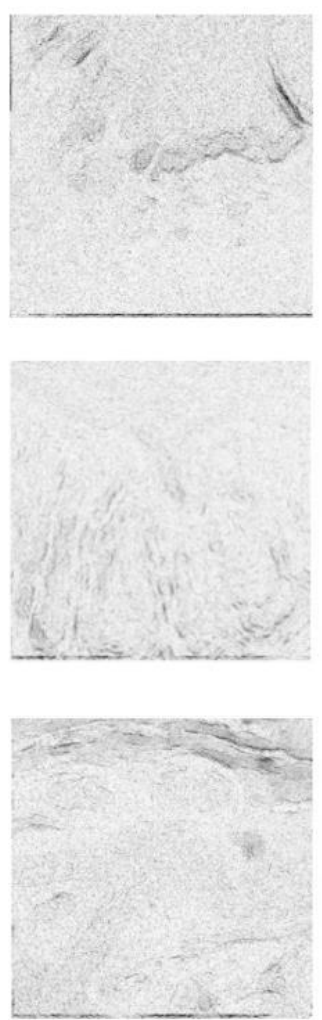

G
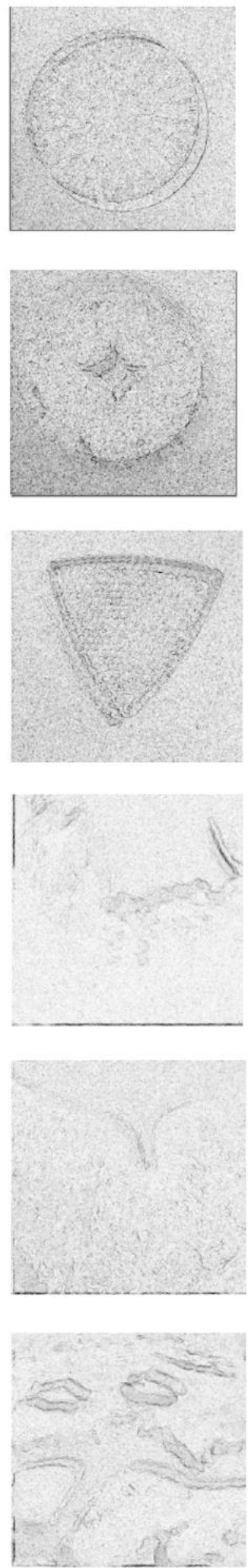

B
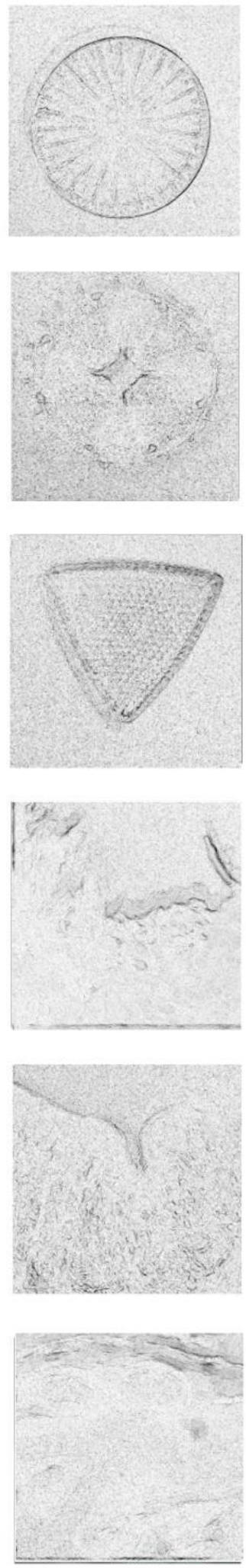

Figure 8. Results of the SSIM test experiment for each pair of images (Chevallier's real image and simulated one). 
TABLE 3. SSIM mean values for each sample image channel.

\begin{tabular}{|c|c|c|c|}
\hline \hline Sample number & SSIM mean R channel & SSIM mean G channel & SSIM mean B channel \\
\hline \hline 1 & 0.9339 & 0.9361 & 0.9381 \\
2 & 0.9323 & 0.9326 & 0.9381 \\
3 & 0.9352 & 0.9310 & 0.9371 \\
4 & 0.9152 & 0.9119 & 0.9038 \\
5 & 0.9109 & 0.9129 & 0.9125 \\
6 & 0.9055 & 0.8876 & 0.8866 \\
\hline
\end{tabular}

\section{Discussion}

This section provides a discussion of the results shown previously and discusses the validity and applicability of the technique used in this study.

\section{5.a. Validity of the method}

Visual inspection of results shown in Figure 7 and Figure 8, and values presented in Table 3 show that the simulated images agree really well with the expected ones generated by the antique microscope objective, indicating that the method used in this study works correctly. In a previous work [10] the basic procedures used in this study were presented and tested by just visual comparison between simulated and real images, and by comparing the calculated PSF and the experimental one captured with a CCD. However, in the present study we present the comparison with a numerical test (the SSIM) for six different sample images. As above commented, a visual inspection shows that the results agree quite well with the expected ones. However, it is important to notice that, from a visual and perceptual point of view, let's say, the "complexity" or the "clutter" of the samples increases from sample 1 to 6. Thus, another test was proposed: to evaluate the validity of the method as a function of the clutter (i.e. the entropy) of the sample images. For that purpose, the complexity of the sample images was computed, by considering the image entropy as follows:

$$
H\left(s_{j}\right)=-\sum_{i=1}^{256} p_{i}\left(s_{j}\right) \log _{2}\left[p_{i}\left(s_{j}\right)\right]
$$

Where $j=1, \ldots, J, H\left(s_{m}\right)$ is the so-called Shannon's entropy [31] of the random variable $s_{j}, p_{i}\left(s_{j}\right)$ the probability of $s_{j}$, which is calculated using the grey level histogram. For that reason, in this final analysis, the sample images were separated in the three different colour channels and then, the entropy $H\left(s_{j}\right)$ of each one was calculated by means of the MATLAB $($ function entropy ( ). Results are shown in Table 4.

TABLE 4. SSIM mean and entropy values calculated for each sample image chan
\begin{tabular}{|c|c|c|c|}
\hline $\begin{array}{c}\text { Sample } \\
\text { number }\end{array}$ & $\begin{array}{c}\text { R channel } \\
\text { entropy }\end{array}$ & $\begin{array}{c}\text { G channel } \\
\text { entropy }\end{array}$ & $\begin{array}{c}\text { B channel } \\
\text { entropy }\end{array}$ \\
\hline \hline 1 & 5.4919 & 5.4762 & 5.6222 \\
2 & 5.7668 & 5.7434 & 5.8446 \\
3 & 5.2672 & 5.2883 & 5.4996 \\
4 & 6.3246 & 6.6748 & 6.6183 \\
5 & 6.7301 & 6.4500 & 6.0019 \\
6 & 5.4919 & 5.4762 & 6.8415 \\
\hline
\end{tabular}




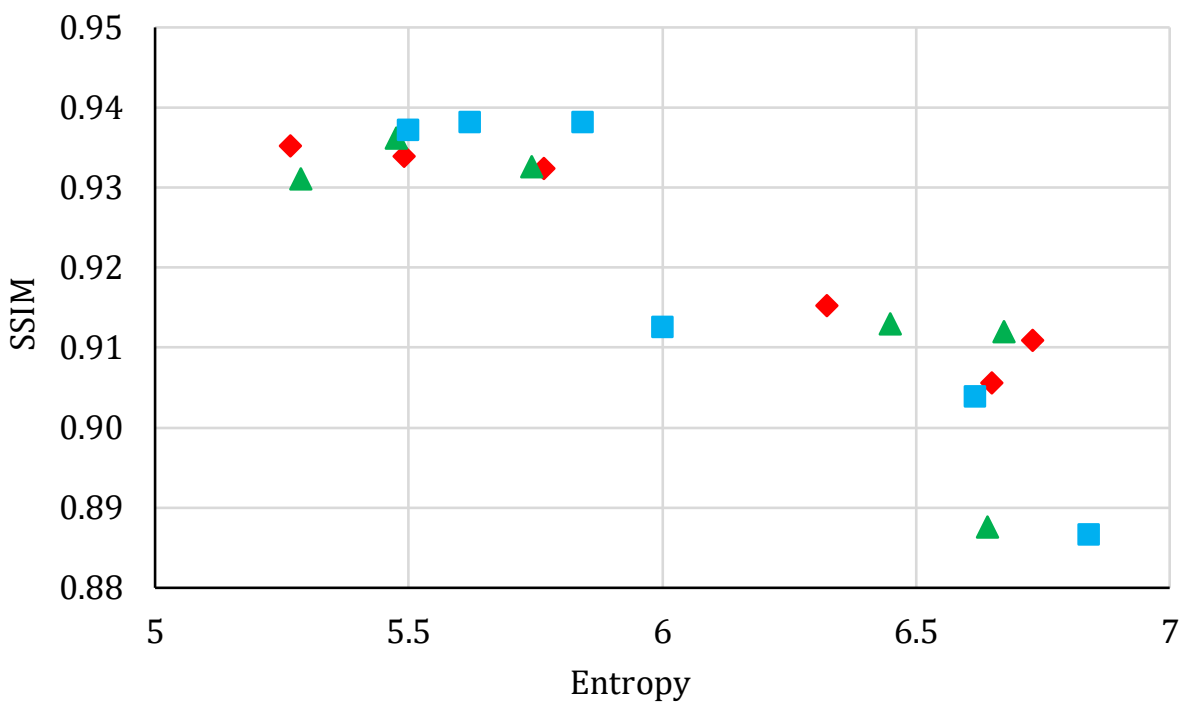

Figure 9. Computed SSIM as a function of entropy per channel for all the samples. The diamond, triangle and square logos are respectively the $\mathrm{R}, \mathrm{G}$ and $\mathrm{B}$ channels of the six images.

As can be seen in Table 4 and Figure 9, the SSIM value per channel apparently decreases when the entropy of the images increases. However, this differences are quite small (see Figure 10) and, taking into account that it was difficult to maintain the same centre of the field-of-view when capturing the experimental images, we can conclude that the technique is valid for any sample images used. Indeed, the effect of this different field-of-view was checked using again the SSIM test, and it was found that it was negligible.

This simple SSIM test together with the results reported in [10] proves the generality and validity of the method used in this work. Moreover, the technique was applied both for telescopes and microscopes, showing that it can be applied to any kind of optical imaging system.

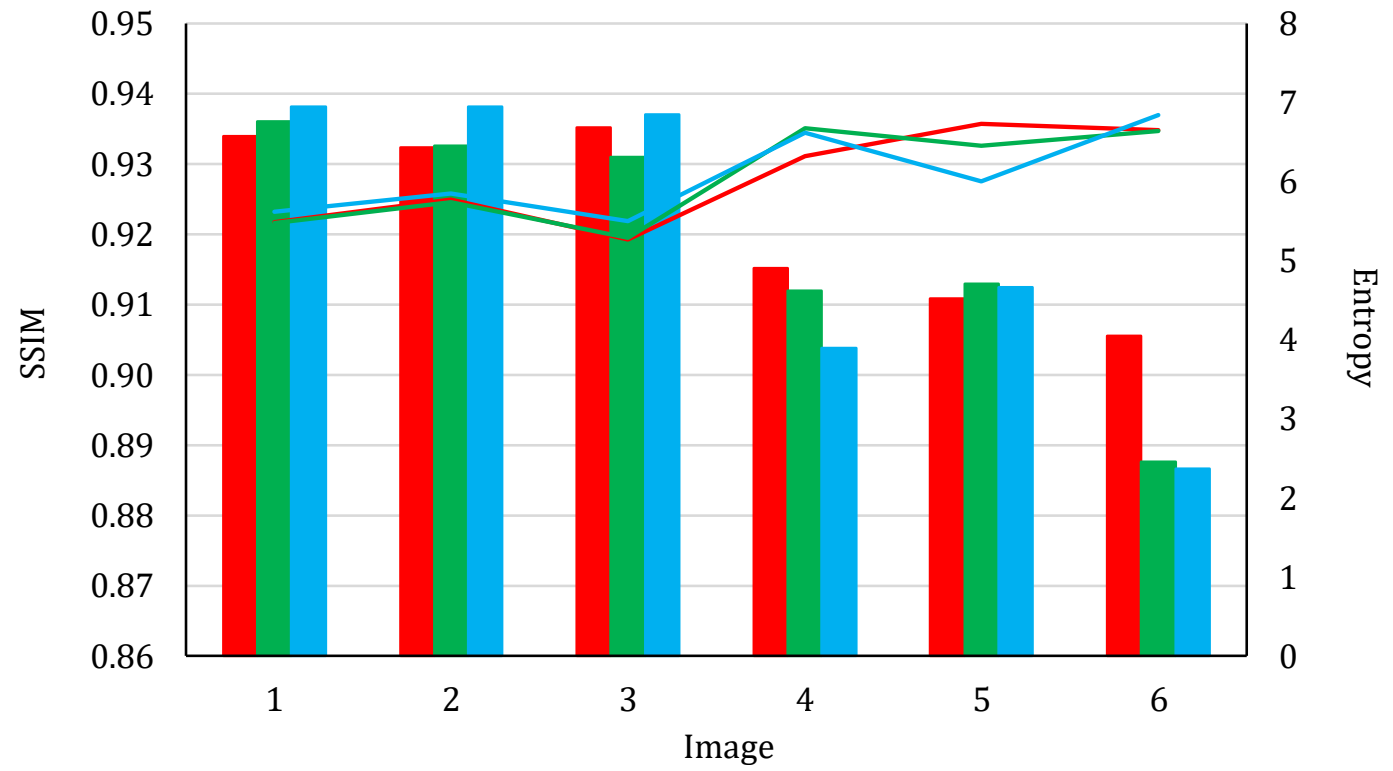

Figure 10. Entropy and SSIM values per sample and channel. The histogram shows the SSIM values for different channel (each group of three different colour columns correspond to a sample), line diagram goes with right axis and show the entropy value per channel. 


\section{5.b. Historical interest of the study and possible future investigations}

The advance and evolution of experimental sciences knowledge is based on the improvement of the measuring instruments and techniques used in every area. Thus, there is a direct correlation with the development of the technologies that provide such instruments and the advance of Science. Particularly, in the fields of Astronomy and Biology, the development and advance of optical techniques and improvement of the image quality of optical instruments has made possible the achievement of fundamental discoveries across the history of humankind. Thus, it results quite evident that the analysis of the optical quality of instruments of different eras can provide a better knowledge on how certain research areas such as Biology or Astronomy have evolved thanks to technological developments. The technique used in this work can be easily implemented to perform such analysis. Moreover, in previous works where the performance and image quality of historical instruments were analysed such as in [32], it was required that the instrument under test had to be removed from the museum and dismantled, which can be dangerous in the case of antique and delicate instruments. We believe that the use of our method can supply the inherent difficulties related to such studies, providing a secure procedure to analyse the instruments, and may add important information to complement studies such the ones presented in [24] and [25].

\section{5.c. Educational potential and interest of this work}

In this work, we have reviewed all the theoretical background necessary to introduce and use the technique presented. The topics presented in Section 2 provide a complete theoretical body of the basis of optical imaging and can be a good complement or introduction to optical design and metrology topics. From the authors own teaching experience those topics covers a huge amount of subjects related with optics and photonics at different levels, as tables 5 to 7 illustrates for the particular cases of the BSc Physics degree and the MSc Photonics degree of the UB and the Universitat Politècnica de Catalunya (UPC) (which is imparted by the UB, the UPC, the Universitat Autònoma de Barcelona (UAB) and the Institut de Ciències Fotòniques (ICFO) together) respectively. Looking at such tables, as an illustrative example of the possibilities of this kind of studies in the Optics and Photonics courses, the topics covered in this work can be related with the different subjects as follows:

- Optics course ${ }^{8}$ at the BSc Physics degree (Table 5): topics 1 and 6, and corresponding laboratory practises [33].

- Image processing and artificial vision course ${ }^{9}$ at the BSc Physics degree (Table 6): topics 1, 3, 7, 10 and 11 [31].

- Photonics laboratory course ${ }^{10}$ at MSc degree in Photonics (Table 7): topics 6, 7, 10 and 11.

Moreover, the technique can be easily adapted as an experimental workshop for advanced undergraduate or master students; even in the case that the laboratory does not dispose of a SH, the Zernike coefficients may be obtained with another technique such as interferometry (as is commonly done [19]), for instance with a Mach-Zender [34] or a Point-Diffraction Interferometer (PDI) as we suggested in [35], or by means of exact ray tracing [7] with commercial or educational programs such as OpticStudio ${ }^{\circledR}$ (ZEMAX ${ }^{11}$ ) or OSLO ${ }^{12}$. These alternatives could provide a cheaper implementation of the experiment at Faculties laboratories. Moreover, the performance of this experiment requires some manual and experimental abilities and programming skills that are interesting for a future photonics or optics engineer. We also believe that the historical motivation and application presented here can be an interesting tool for teaching

\footnotetext{
8 The complete program and information of that course can be found in the corresponding section in: http://grad.ub.edu/grad3/plae/AccesInformePDInfes?curs=2020\&assig=360576\&ens=TG1035\&recurs=pladocent\&n $\underline{2=1 \& i d i o m a=E N G}$. Reference [33] provide a detailed information of the laboratory works.

9 The complete program and information of that course can be found in the corresponding section in: http://grad.ub.edu/grad3/plae/AccesInformePDInfes?curs=2020\&assig=364286\&ens=TG1035\&recurs=pladocent\&n $\underline{2=1 \& i d i o m a=E N G}$. Part of the main theoretical basis within this course is detailed in reference [31].

10 The complete program and information of that course can be found in the corresponding section in: https://photonics.masters.upc.edu/en/curriculum-2020-21. As far the students must choose just three workshops between the different practices, the "topic number" shown in Table 7 does not correspond to the real order of the practices and is just introduced for easy reference in the discussion above. By the time this article is published the program has changed subtly from the one shown in Table 7.

11 OpticStudio $囚$ is a registered trademark from Zemax@ $\subset$, commonly used for ray tracing and optical design.

12 OSLO ${ }^{\circledR}$ states for Optics Software for Layout and Optimization, and it is available under an educational and three commercial versions from Lambda Research Corporation`C.
} 
these subjects, given that it has its own value as a tool to offer new perspectives and information for research in history of science and technology.

TABLE 5. Program of the compulsory course of Optics of the BSc degree in Physics at the UB.

\begin{tabular}{|c|c|c|}
\hline $\begin{array}{l}\text { Topic } \\
\text { number }\end{array}$ & Theoretical block & Laboratory practises \\
\hline 1 & Optical instruments & $\begin{array}{l}\text { Design and construction of } \\
\text { optical instruments } \\
\text { Spectrometry and chromatic } \\
\text { dispersion }\end{array}$ \\
\hline 2 & $\begin{array}{l}\text { Light propagation in vacuum. } \\
\text { Polarization phenomena }\end{array}$ & $\begin{array}{l}\text { Polarization of light (Malhus } \\
\text { law) }\end{array}$ \\
\hline 3 & $\begin{array}{l}\text { Light propagation in dielectric and } \\
\text { conductive media }\end{array}$ & $\begin{array}{l}\text { Reflection of light in dielectric } \\
\text { media (Brewster's angle) }\end{array}$ \\
\hline 4 & $\begin{array}{l}\text { Interference phenomena and } \\
\text { applications. Laser }\end{array}$ & $\begin{array}{l}\text { Young interferences: } \\
\text { Fresnel's biprism } \\
\text { Michelson interferometer }\end{array}$ \\
\hline 5 & Diffraction. Fourier Optics & Diffraction \\
\hline
\end{tabular}

TABLE 6. Program of the elective course of Image processing and artificial vision of the BSc degree in Physics at the UB.

\begin{tabular}{|c|c|}
\hline Topic number & Theoretical block \\
\hline 1 & Fundamentals of image processing \\
\hline 2 & $\begin{array}{l}\text { Basic operations for image manipulation: rotation, } \\
\text { interpolation, resolution changes, zoom. Cameras }\end{array}$ \\
\hline 3 & $\begin{array}{l}\text { Intensity transformation: contrast changes, histogram, } \\
\text { equalization. Color spaces }\end{array}$ \\
\hline 4 & Security: steganography and encryption \\
\hline 5 & Clustering: k-means \\
\hline 6 & Classification (kNN). Support Vector Machines (SVM) \\
\hline 7 & Fourier Transform \\
\hline 8 & Spatial filtering \\
\hline 9 & Filtering in the frequency domain \\
\hline 10 & Image formation in optical systems. PSF and OTF. Defocusing \\
\hline 11 & Image restoration techniques \\
\hline
\end{tabular}


ÓPTICA PURA Y APLICADA

www.sedoptica.es

TABLE 7. List of laboratory practices with the Photonics laboratory compulsory course of the MSc degree of the UPC.

\begin{tabular}{|c|c|c|}
\hline Topic number & Laboratory work & Responsible University \\
\hline 1 & $\begin{array}{l}\text { Interference in amplitude division devices. } \\
\text { Nonlinear effects in a Michelson interferometer }\end{array}$ & UAB \\
\hline 2 & Polarization and polarizing materials & $\mathrm{UAB}$ \\
\hline 3 & $\begin{array}{l}\text { Light-matter interaction phenomena. The } \\
\text { Zeeman Effect }\end{array}$ & UAB \\
\hline 4 & Atomic spectroscopy. Optical pumping & UAB \\
\hline 5 & $\begin{array}{l}\text { Mutual Intensity. Measurement of degree of } \\
\text { spatial coherence }\end{array}$ & UB \\
\hline 6 & $\begin{array}{l}\text { Fractional Talbot images generation by Fresnel } \\
\text { diffraction }\end{array}$ & UB \\
\hline 7 & $\begin{array}{l}\text { Dealing with resolution and magnification: } \\
\text { telescopes and microscopes }\end{array}$ & UB \\
\hline 8 & $\begin{array}{c}\text { Active and nonlinear optical media: lasers and } \\
\text { harmonic generation }\end{array}$ & UPC \\
\hline 9 & $\begin{array}{l}\text { Photoemitters and photodetectors. Distance } \\
\text { measurements with laser }\end{array}$ & UPC \\
\hline 10 & Optical image processing & UPC \\
\hline 11 & Hands-on image sensors & UPC \\
\hline 12 & Optical fibers: hands-on and characterization & UPC \\
\hline 13 & Optical fiber transmission: network and devices & UPC \\
\hline 14 & $\begin{array}{l}\text { Optical fiber communication systems: Erbium } \\
\text { Doped Fiber Amplifiers }\end{array}$ & UPC \\
\hline
\end{tabular}

\section{Conclusions}

We have presented a method based on wavefront sensing for analysing antique optical instruments and we have presented a study of the optical performance of an old microscope objective using such technique. The method used in such analysis was tested with different RGB images, providing results that agree within the theoretical framework. Furthermore, the potential use of such method for educational purposes had been pointed out in the context of the syllabus of three different courses for undergraduate and master's students in which the authors have teaching experience.

Furthermore, the study of antique scientific instruments makes it possible to appreciate the limitations of scientific research at any particular era. Particularly, the analysis of image quality of antique optical instruments permits to correlate it with discoveries and advances in scientific areas such as Astronomy and 
Biology. We believe the use of non-invasive techniques such as the one presented here could add new information to historiographic analysis in different scientific topics.

\section{Acknowledgements}

This work has been possible thanks to the works carried out within the projects HAR2008-02580-EHIST, DPI2008-04175/DPI and FIS2016-77319-C2-2-R.

The instrument analysed belongs to the Collection of Scientific Instruments of the Faculty of Physics of the UB and to the Museu Virtual of that university. 